\title{
A Literature Review of Coding for Early Childhood
}

\author{
Reza Edwin Sulistyaningtyas ${ }^{1}$, Prasetyo Yuliantoro ${ }^{2}$, Dian Astiyani ${ }^{3}$, Chomisah Nugraheni ${ }^{4}$ \\ \{reza.edwin@unimma.ac.id ${ }^{1}$ \}
}

Early Childhood Education, Universitas Muhammadiyah Magelang, 56172, Magelang 1,3,4

Telecommunication Engineering, Institut Teknologi Telkom Purwokerto, 53147, Purwokerto ${ }^{2}$

\begin{abstract}
Since coding is an essential skill in the 21st century, computational thinking should be built first as its foundation. Introduction to coding can start from an early childhood and early level of elementary school. This article systematically searched for some articles about coding for early childhood. A total of nine articles discussing coding for early childhood, the tools used, and its influences on early childhood development were analyzed. These articles were selected based on year of publication, age group, and learning outcome. The results found that the introduction of coding for early childhood can be implemented through plugged-in and unplugged activities. The literature revealed that coding on early childhood development can increase cognitive ability, communication, collaboration, creativity, and executive function (planning, task completion, and inhibition decrease).
\end{abstract}

Keywords: Coding, early childhood, computational thinking, literature review

\section{Introduction}

Early childhood has recently been exposed to a technologically savvy environment (mobile phone, computer, refrigerator, automatically operated door, etc.). Along with the rapid growth of technology from time to time, the world of education has also utilized technology as a learning medium. For example, school-age children have currently used mobile phones and laptops to find important information for supporting the learning process or for the sake of entertainment such as playing games. However, most children only know how to use it without knowing how the program runs the devices inside. The knowledge, understanding, and skills of digital technology, such as coding literacy, will form the basis of the digital literacy practices that will be useful for children at school [1]. For these purposes, most of the current school curriculum includes computer programming activities as additional programs.

Indeed, computer programming is one of the must-have skills in the 21st century [2]. Computer programming is a list of step-by-step instructions for the computer [3]. Learning computer programming or coding can be introduced from an early age. Bers [4] stated two coding frameworks: 1) coding as a playground of an environment designed to stimulate all aspects of development and coding as literacy defined as the ability to use symbols and technological tools to understand, produce, communicate and express ideas or thoughts by creating products that can be shared. Nonetheless, there is still much unknown to educators and product developers as to what skills children will develop as they code even as computer programming for early childhood becomes increasingly popular [5]. Coding practices involve a variety of early math skills and scientific processes, including spatial ability, numeric 
comprehension, problem solving skills, inquiry skills, and reasoning skills [6]. Coding practices also need the involvement of capabilities in computational thinking.

The term "computational thinking" refers to analytical processes rooted in computer disciplines and programming activities [2]. Computational thinking is divided into three computational concepts (testing \& debugging), computational practice (problem solving) and computational perspective (relationship between child and digital world) [7][8]. According to Muñoz-Repiso \& Caballero-González [9] there are three dimensions to measure computational thinking skills including sequences (algorithms), action-instruction correspondence and debugging. According to Piaget's Theory of Cognitive Development, computational thinking skills that can be introduced to preschoolers are pattern recognition and algorithm design [10].

The introduction of coding in early childhood and primary school education cannot be separated from the role of teacher. However, the teacher's understanding of the introduction of coding is challenged with various obstacles including 1) the misunderstanding in the concept of computational thinking for some teachers; 2) lack of knowledge as the main challenge of teachers in teaching coding, i.e. pedagogical content knowledge and content knowledge 3) the influence of undeniable non-cognitive factors, such as anxiety, self-confidence, on the teachers' teaching ability;4) the important role of school culture involvement [11].

The application of coding at the education level of early childhood raises a variety of questions. Therefore, this literature review study was aimed to answer the following questions:

1. How is the application of coding in early childhood education?

2. What is the impact of coding on early childhood development?

\section{Method}

This article collected data on coding research studies for early childhood published from 2015 to 2020. The databases used for the search included Google Scholar, EBSCOhost, SAGE Journal, ProQuest \& Taylor \& Francis. Keywords used to search were: "coding", "computer program", "computational thinking" combined with "early childhood", "kindergarten", "preschool", "early years", and "first grade". The important thing on record is that the age of early childhood refers to the NAEYC (National association for the Education of Young Children) which is $0-8$ years old. Therefore, early primary school age children who belong to early childhood are included in the search.

\section{Results and Discussion}

This section will review the research on coding for early childhood (2015-2020). A total of 9 research papers were selected based on the predetermined criteria. Descriptive statistics were displayed to provide a clear picture of the studies that have been collected based on the year of publication, population sample, method, technology used, and learning outcome.

\subsection{Unplugged activities}

The introduction of coding in early childhood through unplugged activity is an activity process without using a computer. Although coding is a relatively new term in early childhood education, children experience and use coding in their daily lives and routines with unplugged 
practices (e.g., learning to tie their shoelaces by following a series of steps). Precoding or early coding is an introductory to coding activities for early childhood. Precoding activities can be done by introducing command languages, such as go up, go down, move backward and move forward, and turn right and left [12]. According to Lee [6], unplugged activity stages include the use of directional words or directional arrows (move forward, move backward, turn left, turn right), using sequential words (first, second, third, etc.), combining both directional and sequential words and connecting with grids. These stages are implemented in children's daily activities, such as hand washing activities. The teacher's job is to ask the child to compose the hand washing algorithm by sorting the images of the hand washing stages from start to finish on the code sheet (See Table 1).

Table 1. Result of Review on Coding Early Childhood ( $\mathrm{N}=9$ )

\begin{tabular}{lll}
\hline \multicolumn{1}{c}{ Variable } & \multicolumn{1}{c}{ Material } & $\mathbf{N}$ \\
\hline \multirow{3}{*}{ Year of the data } & $2015-2016$ & 1 \\
& $2017-2018$ & 4 \\
Sample population & $2019-2020$ & 4 \\
& Kindergarten & 4 \\
Method & First grade & 1 \\
& Mix method & 2 \\
Experiment & 3 \\
Technology used & Literature review & 4 \\
& SctratchJr & 4 \\
& TurtleBot & 1 \\
& Code.org & 2 \\
& KIBO robotics kit & 1 \\
& SctratchJr \& KIBO robotics kit & 1 \\
& Problem solving & 1 \\
& Cognitive abilities & 1 \\
& Children's planning and inhibition skills & 1 \\
\hline
\end{tabular}

Recently, robotics and computer programming have become a focus by kindergarten teachers as a method for teaching academic skills to kindergarten children through hands-on experience with new technologies [13]. Saxena et al [10] mentioned that unplugged activities to improve computational thinking skills as the basis for coding include the command through Bee-Bot (robots that can be inserted with sequential programs to execute commands), LEGO bricks (activities to learn pattern recognition, examples of orange-blue-orange-blue) and sequence series (composing in order of daily activities). Another toy tool that can be used is KIBO. KIBO is a screen-free robot kit specifically designed for children ages 4-7 years old. Children can build their own robot, program it to do what they want, and decorate it with art supplies [14]. There is also TurtleBot, which is a programmable robot by scanning cards sequentially [13]. The green card is for the forward command, the red card is for the backward command, the blue card is used to make the robot turn right, and the yellow card is used to make the robot turn left.

\subsection{Plugged-in activities}

Plugged-in activities are the process of introducing coding in early childhood using computers. Duncan, Bell \& Tanimoto [15] classified the level of Initial Learning Environments 
(ILE) coding for children from levels 0 to 4 . The level for early age is at level of 0 (Age range of 2-7 years including drag-and-drop or simpler, teaching planning/sequence only, \& requiring no abstraction) and Level 1 (Age range of 5-10 years, including drag-and-drop, \& requiring no abstraction). Tools at levels 0 and 1 to introduce coding through plugged-in activities in early childhood are ScracthJr and Code.org.

ScracthJr is a learning tool to introduce the concept of computer programming to children aged 5 to 7 years [16]. ScratchJr enables children to create interactive stories and games by snapping together graphical programming blocks to make characters move, jump, dance, and sing [14]. Programming blocks are made as an interesting activity and displayed with a variety of sample colors (e.g. blue blocks to move up, down, right and left). These programs can range from very simple activities (e.g. one character increases in size) to quite complex (e.g. two or more characters interacting and chatting in dynamic settings) [5]. Another tool that can be used is code.org. Code.org is a non-profit organization that particularly aims for school students in teaching computer science [17]. Students can easily form a string of commands by clicking and then dragging blocks in code.org.

\subsection{The impact of coding on early childhood development}

The research conducted by Çiftci \& Bildiren [18] showed that the coding course had a positive effect on the non-verbal cognitive abilities of the participating children, but the problem-solving skills were statistically insignificant. In contrast to Arfe's results, Vardanega \& Ronconi [19] stated that coding not only improved children's ability to solve problems, but also improved executive functions (spending time for planning, solving standardized planning tasks, and inhibiting prepotent responses). In addition, the introduction of coding from an early age can support communication, collaboration, and creativity in the classroom [20].

\section{Conclusion}

Coding is becoming a new literacy in the 21st century that can be introduced from an early childhood and early primary school along with the current development of digital technology that has been inseparable from our surroundings. The currently rapid development of technology requires us to prepare human resources in order to be able to create these technologies. Mastery of computational thinking, problem solving skills, and gradual thinking are the capabilities that must be mastered to perform coding. In addition, there needs to be a strategy to introduce coding in early childhood through plugged-in and unplugged activities. The activity encourages early childhood and early primary schoolers to learn about coding in an interesting and fun way. Plugged-in activities that use tools on the computer include ScratchJr and Code.org. Unplugged activity refers to some activities without using a computer, including sorting images of daily activities from start to finish, getting to know directions, and playing with LEGO bricks and robots (KIBO, Bee-Bot, and TurtleBot). There are not many papers to research on coding for early childhood, but some existing papers have proven that the introduction of coding for early childhood can encourage a variety of children's abilities, including cognitive abilities, executive function, problem solving, communication, creativity, and collaboration. On this basis, it is suggested that further research conducts some research on the effectiveness of unplugged and plugged-in activities to introduce coding to early childhood. 


\section{References}

[1] C. Walsh and C. Campbell, "Introducing Coding as a Literacy on Mobile Devices in the Early Years," in Introducing Coding as a Literacy on Mobile Devices in the Early Years, 2018, pp. 51-66.

[2] M. U. Bers, "Coding as another language: a pedagogical approach for teaching computer science in early childhood," J. Comput. Educ., vol. 6, no. 4, pp. 499-528, 2019.

[3] K. Clausen, "A Pre-Coding Primer for Preschoolers," Child. Libr., vol. 17, no. 4, pp. 19-21, 2019.

[4] M. U. Bers, "Coding, Playgrounds and Literacy in Early Childhood Education: the Development of KIBO Robotics and ScratchJr Marina," in 2018 IEEE Global Engineering Education Conference (EDUCON), 2018, pp. 2100-2108.

[5] A. Strawhacker and M. U. Bers, What they learn when they learn coding: investigating cognitive domains and computer programming knowledge in young children, vol. 67, no. 3. Springer US, 2018.

[6] J. Lee, "Coding in early childhood," Contemp. Issues Early Child., vol. 21, no. 3, pp. 266-269, 2019.

[7] S. Y. Lye and J. H. L. Koh, "Review on teaching and learning of computational thinking through programming: What is next for K-12?," Comput. Human Behav., vol. 41, pp. 51-61, 2014.

[8] H. Palmér, "Programming in Preschool--With a Focus on Learning Mathematics.," Int. Res. Early Child. Educ., vol. 8, no. 1, pp. 75-87, 2017.

[9] A. G. V. Muñoz-Repiso and Y. A. Caballero-González, "Robotics to develop computational thinking in early Childhood Education," Comunicar, vol. 27, no. 59, pp. 63-72, 2019.

[10] A. Saxena, C. K. Lo, K. F. Hew, and G. K. W. Wong, "Designing Unplugged and Plugged Activities to Cultivate Computational Thinking: An Exploratory Study in Early Childhood Education," AsiaPacific Educ. Res., vol. 29, no. 1, pp. 55-66, 2019.

[11] R. Kong and G. K. W. Wong, "Teachers' perception of professional development in coding education,” in 2017 IEEE International Conference on Teaching, Assessment, and Learning for Engineering (TALE), 2017, pp. 377-380.

[12] D. P. Mclennan, "Creating Coding Stories and Games," Teaching Young Children, 2017.

[13] K. W. Nam, H. J. Kim, and S. Lee, "Connecting Plans to Action: The Effects of a Card-Coded Robotics Curriculum and Activities on Korean Kindergartners," Asia-Pacific Educ. Res., vol. 28, no. 5, pp. 387-397, 2019.

[14] M. U. Bers, "Coding and Computational Thinking in Early Childhood: The Impact of ScratchJr in Europe,” Eur. J. STEM Educ., vol. 3, no. 3, pp. 1-13, 2018.

[15] C. Duncan, T. Bell, and S. Tanimoto, "Should your 8-year-old learn coding?," in Proceedings of the 9th Workshop in Primary and Secondary Computing Education, 2014, pp. 60-69.

[16] A. Strawhacker, M. Lee, C. Caine, and M. Bers, "ScratchJr Demo: A coding language for Kindergarten Amanda," in Proceedings of the 14th International Conference on Interaction Design and Children, 2015, pp. 414-417.

[17] U. T. Kaplancali and Z. Demirkol, "Teaching Coding to Children: A Methodology for Kids 5+," Int. J. Elem. Educ., vol. 6, no. 4, pp. 32-37, 2017.

[18] S. Çiftci and A. Bildiren, "The effect of coding courses on the cognitive abilities and problem-solving skills of preschool children," Comput. Sci. Educ., vol. 30, no. 1, pp. 3-21, 2019.

[19] B. Arfé, T. Vardanega, and L. Ronconi, "The effects of coding on children's planning and inhibition skills,” Comput. Educ., vol. 148, pp. 1-16, 2020.

[20] M. U. Bers, C. González-González, and M. B. Armas-Torres, "Coding as a playground: Promoting positive learning experiences in childhood classrooms," Comput. Educ., vol. 138, pp. 130-145, 2019. 